\title{
Fictional Proto-architecture as an Introduction to Biologic Design: Challenging the Concept of Morphogenesis of Neo-architectural Organism
}

\author{
Andreea Felciuc ${ }^{a^{*}}$ \\ ${ }^{a}$ Architect RIBA, ARB, PhD Candidate and Research Fellowship, Architecture Doctoral School (SDA), "Ion Mincu" University of \\ Architecture and Urbanism, Bucharest, Romania
}

\begin{abstract}
The architecture is based on a dialectical search for new ways of matter representation. We deal with the form of contemporary architecture under two approaches: expression and content. The article examines how mathematical principles based on natural growth can be applied in architectural design to create a dynamic, not static, structure. The dynamic process of the cell and its growth provides the basic structure. The continuity of the domain is exemplified by the impact of the new forms on the society that has already begun to emerge from the obscurity. The paper argues that without a deeper and more receptive connection between geometry and performance from a bio-morphogenetic perspective of complex systems. The experimental design methods are applied both to generate and to evaluate an architecture of the futuristic lines. These methodological frameworks focus on cyclically restated themes in the field of parameterises, which are identified as endemic to architecture: the realization of buildings, of multifunctional volumes and customized per se through a gradual approach of the architectural properties and the exploitation of a "concept construction" integrated as a process, obtained through innovative modelling environments. And so, and the reconstruction of architecture as an organ of nature is demonstrated. The new vanguard of proto architecture describes difficulties and inconsistencies in the relationship between theories and structures, difficulties arising from the very idea of "virtually" itself. It becomes difficult to say that a drawing in cyberspace is an architectural form or just a graph of architectural theory; in the virtual space, there is no difference between the particular structure and the general principle. Therefore, the form is first designed, only after to be constructed. Naturally, it is impossible (theoretically or technically) for design and construction processes to take place simultaneously. Predictably, bio-morphosis leads to multiple forms of expression, defined and transmitted in geometric terms.
\end{abstract}

\section{Keywords:}

Self-referential Form;

Phenotypic Plasticity;

Reaction Norms;

Resilience;

Minimal Organism.

\section{Article History:}

$\begin{array}{llll}\text { Received: } & 21 & \text { June } & 2020 \\ \text { Accepted: } & 22 & \text { November } & 2020 \\ \text { Published: } & 01 & \text { December } & 2020\end{array}$

\section{1- Introduction}

The emergence of innovative developments in the field of architecture and generative design, biology, microbiology, biotechnology, biomimetic, morphogenesis of medicine and nanotechnology defines inclusion of an essential definer of the new architectural paradigm, requiring focus on the development of a new methodological framework with apparent implications. Aesthetic and technical. This paper investigates the impact of these emerging and progressive biological advances on architectural and design practices. Within the current background of experimentalizing and hybridization that uses design as a method of exploration and manipulation of current biological material, the notion of proto-design and proto-architecture appears as an interdisciplinary, biomorphogenetic working methodology, creating technology and a design: hybrid, new materiality and potentially unprecedented forms of life [1,2].

\section{* CONTACT: Andreea.felciuc@ andreea-felciuc.com}

DOI: http://dx.doi.org/10.28991/esj-2020-01248

(C) 2020 by the authors. Licensee ESJ, Italy. This is an open access article under the terms and conditions of the Creative Commons Attribution (CC-BY) license (https://creativecommons.org/licenses/by/4.0/). 
The results of these conditions, hereafter defined as "architectural proto-design" represent assemblies of objects partially designed and partly living organisms. The natural/artificial delimitation is progressively extinguished to a complete delineation. This new area of research is shown to be more than derived from extended analogies between bio-morphological conditions (cellular structures) and human-scale forms (architecture), as they are commonly expressed in current bio-architectural works. Proto architecture involves architectural organisms consisting of two entities: organic and inorganic [3-6].

The experimentation of hybrid design through the generation of bio-architectural compositions requires the definition of a new and innovative complex methodological framework [7]. In this context, the architectural expression must be considered a concept that presents itself in a state of continuous development, encompassing a variety of generative experiments that cannot be assimilated as a coherent and comprehensive organism. Formal investigations, more than bodies (an attribute historically defined as a distinct functional apparatus) [8] or assemblies (performance-driven and digitally generated geometric systems), represent bio-morpho-genetic composites of architecture that sometimes appear as constructed or otherwise defined entities appear instead as living beings [9-11].

Proto-architecture is under the auspices of a phenomenon defined as a sum of current terminology: biomorphogenesis, biomimetic, parametric, biophilic, organic architecture, biomimicry, machine learning. There are only a few people who form the language of the new paradigm, a common terminology of a transdisciplinary topic [12, 13]. However, hybrid and experimental architecture continues to be perceived as a domain that is fundamentally removed from all such phenomena, primarily when understood as a discipline that does not exclusively concern the built environment per se.

Proto architecture is based on parametric, biomimetic and morphogenetic principles but differs and deviates from them. The position adopted by this new area of research, from specific perspectives, differs critically; researches an emerging territory that explores current biological generation practices and their implications for the field of architecture [14].

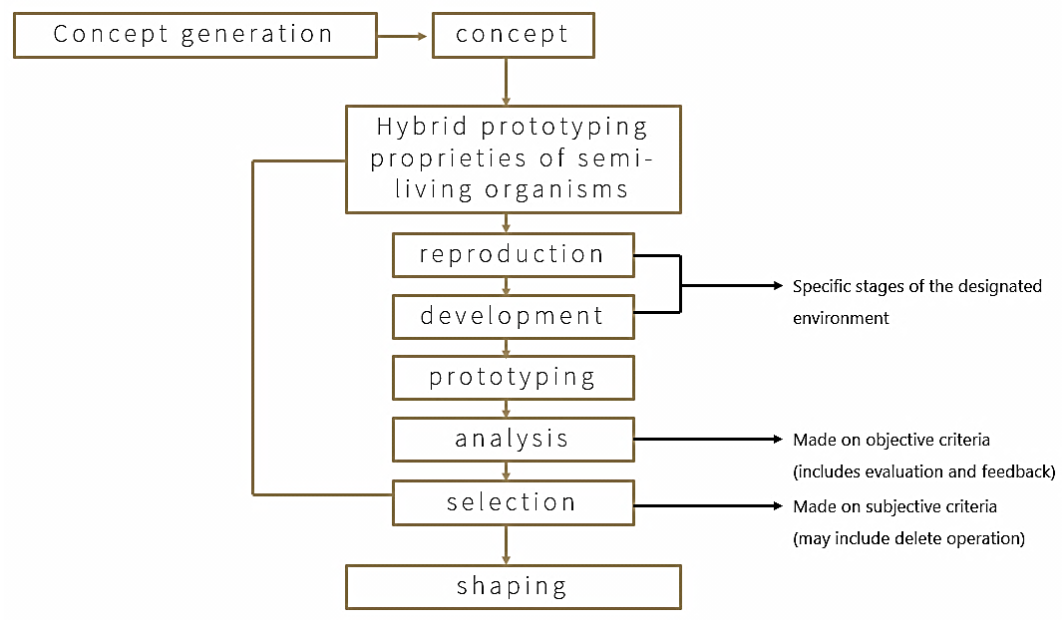

Figure 1. Operations that define the process of the study, creating criteria and hierarchies of shapes/structures/volumes.

The principles found in organic architecture defined by biology and morphogenesis were used as a definition of the design concept. Biomorphic architecture, as defined by Gunter Feuerstein, is an endorsement of the anthropomorphic principles applied in the design and design, assuming the establishment of a formal link between nature and architecture. Current research suggests that architectural construction functions as a living organism, and therefore could be organized to respect principles, operations, laws and processes similar to those governing living organisms and systems $[15,16]$. The study of biological phenomena by translating in morphological terms and applying the principles of these phenomena as a means of developing new structural and formal methods includes biotechnology, genetics, molecular biology, biochemistry, embryology and cell biology, as well as information technology, robotics, sophisticated mathematics and geometry. Advanced, parametric generated modelling and modelling. The availability of transgenic organisms and the parameterization of the architecture denote a hybridized tendency in proto architecture [17]. The practice of experimental design includes the biological and medical research laboratory as a prolific workshop environment to use the terminology specific to these disciplines as a generating principle and a systematic architectural process. Exploring the potential aesthetic impact of imaging created by medical or laboratory equipment, as well as including primary or advanced geometric operations, represent proto-hybrid design tools [18]. Biology and geometry have become a new plastic environment, being tools for expressing the architectural gestures defined by a non-Cartesian approach to biology and medicine. We define a prolific framework for designing and creating new living conditions. 
The physical context in which the new architectural body will be implanted will become increasingly infused with "principles of bio-logic" (Kevin Kelly) merging "projected technology and nature to agglutination". Thus we place ourselves unmistakable to a paradigm shift, in which we outline a future neo-proto-biological era, in which architecture will exist through organisms that move living silicon polymers, software programs that evolve offline, adaptable and modular machines, spaces which includes coevolutionary pieces of furniture, robots and artificial intelligent entities, biologically manufactured viruses that have vindictive properties, neuronal synapses, mechanized body parts, simulated personalities as well as a vast ecology of computing devices in constant flux [19].

However, no matter how utopian these descriptions may be, the proto-architectural design does not imply defining a completely innovative vision, in which architecture is entirely replaced by neo-biological equipment and workshops, but rather by an evolutionary and emergent scenario [20]. In which the pre-existing, possibly vernacular context will be inserted, implanted by it, creating new hybrids and complex systems of architectural bodies. Research, rather than presenting predictive views, defines challenges by creating debates beyond parametric paradigms, which are not based purely on geometrically driven discourses in the digital domain [21].

Proto-architectural design represents the conviction of changes that inevitably occur in architecture and which require understanding beyond the traditional disciplinary boundaries, not only of how we understand the interaction between us and the space in its natural habitat, but also how architecture responds to architectural objects hybridized with biological matter, which creates semi-living systems described by an unpredictable nature [22]. The current architectural practice is critically challenged, not in terms of the instrumentation used as the basis of the design language, expertise and body of knowledge and emerging interdisciplinary work methodologies, but also in terms of its aesthetic intent. The new architectural body is defined by the ability to avoid some constraints imposed by the inheritance of a formal aesthetic that has affected architecture so profoundly during the last century.

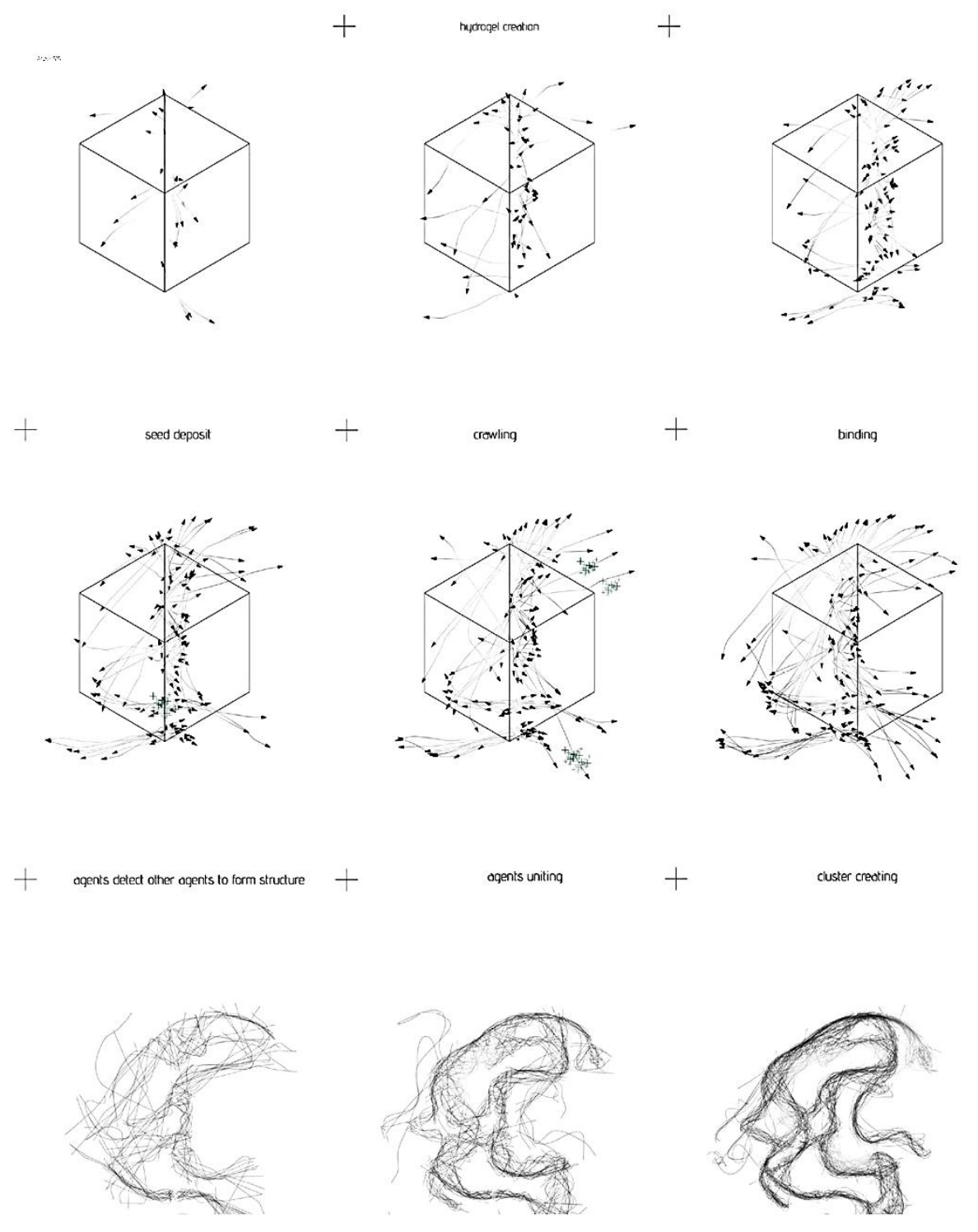

Figure 2. Structural stabilization of the spatial organization. 
The current research segment is defined by new terms, taken from biology, as follows: "morphogenesis", "hydronics" "homeostasis"; which once introduced into the current design practice have far-reaching etymological implications on architectural language. Proto architecture as a design methodology embraces the attempts of emerging concepts, self-organization, self-assembly and self-replication, so structural bodies begin to be confused with biological organisms. In this context, hybridized design continues to be crucial in testing new phenomena and in developing a new vocabulary that articulates the potential of new semi-living architectural systems and processes, these "being-objects" that evolve in life, in part [23].

The research is based on ideas and projects often rejected as being too speculative or design or design hypotheses. Architecture is defined as a body without organs, becoming a relevant topic considered as the way of controlling, maintaining and sustaining living conditions, of handling and managing micro-organic matter in architecture, as well as growing semi-living structures. The role of hybrid design within the development and integration of devices, equipment, methods of supervision, support systems, prostheses and hinges are to enable the growth of the architectural body and helps them to develop in an architectural context that has significant relevance [24-26].

The practice of proto-architecture proposes the exploration of new minimal geometry, of a surface with fundamental characteristics integrated within the development of a new paradigm, as well as a new hybrid methodology and the use of advanced visualization and 3-D modelling software in both biological sciences, as well as in architecture [27]. Finally, the significance of morphogenesis procedures and new biomaterials is discussed, combined with the potential to facilitate the emergence of a modern aesthetic, defined to some extent by the reconfigured language necessary to express the proto-architectural design.

\section{2- Adaptive Cinematic and Scenic Morphologies}

The kinetic morphologies of the proto-architecture adaptive to the performing arts are based on the analysis of the development of the performing spatial organizations and on implementation methods on the scenic structure and the existing projected space [28]. The paper proposes an analysis of these methods, biological systems and models that can provide a basic framework for further development, ideas and strategies that can serve as a starting point for the development of the organization of hybrid proto-architectural bodies within the performative architecture.
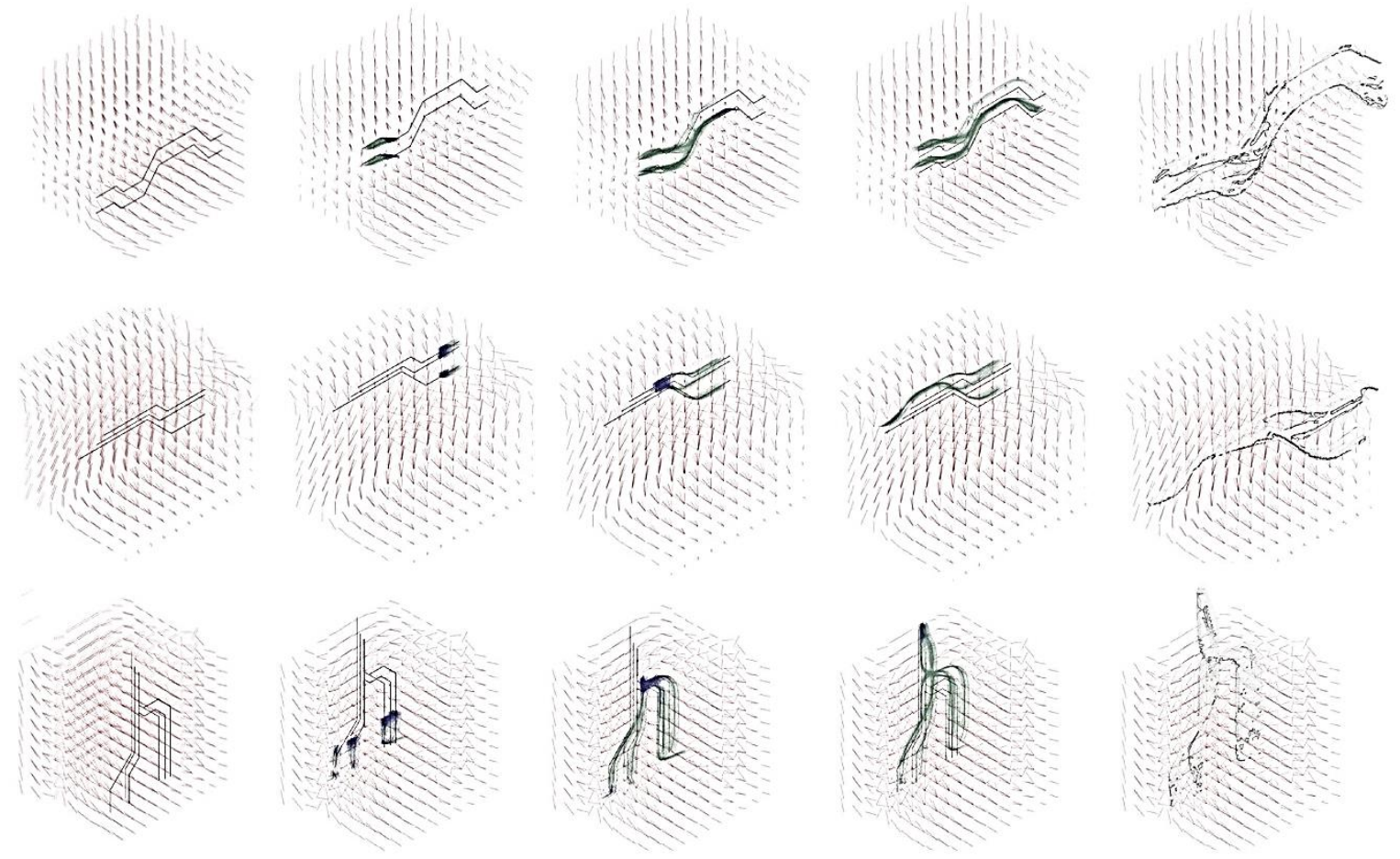

Figure 3. Proto-typing the architectural body - the interaction of the sub-systems.

The research aims to identify specific organisms or biological organizations that have a high potential for the acquisition of structural and formal architectural features through their adaptive performance and successive transformation methods [29]. The analysis summarizes the processes underlying their metamorphosis and describes systems of digital geometric models that lead to their development into performative prototypes whose material and formal properties allow speculation about their architectural applicability. 
2-1- Criteria: Research in the Field of Biology to Identify Phenomena of Possible Biological Organisms Whose Interactivity Affects Adaptive, Participatory and Interactive Performance

- Types of linear or surface transformation: stretching, closing, unfolding, inflating;

- Opacity vs. transparency;

- Closed forms/organisms against perforated or open;

- Changes in materials and textures;

- Changes in surface qualities and their articulation (smooth to articulated);

- Types of reactions and responses to stress, exposure, humidity, temperature;

- Reactions in contact with other biological organisations.

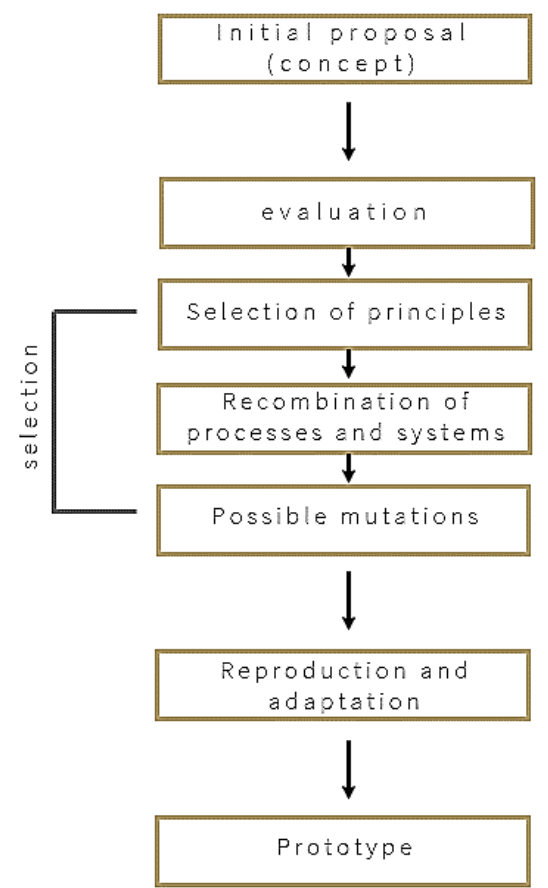

Figure 4. Ways in which the shape can regenerate, grow and / or adapt to the environment in which it is placed.

Each of these systems, explaining the nature and type of their metamorphosis, is analysed and the underlying biological processes and syllogisms that guide and facilitate the respective transformations are identified and defined. Why is it transforming? How is it transformed? [30].

\section{2-2- The Phase of Abstraction and Translation}

From the phenomenon to the system: The phenomena previously identified will be carried out in the second research phase. The morphological, tectonic and geometric characteristics and properties of the phenomena in question are identified and abstracted: the transition from the biological domain to an abstract digital environment. Digital kinetic models and animations are developed to simulate and test their systematic, tectonic and geometric transformations and limits within the logic of abstract systems [31]. The resolved digital forms and models do not yet contain any information about functions, materials or scales.
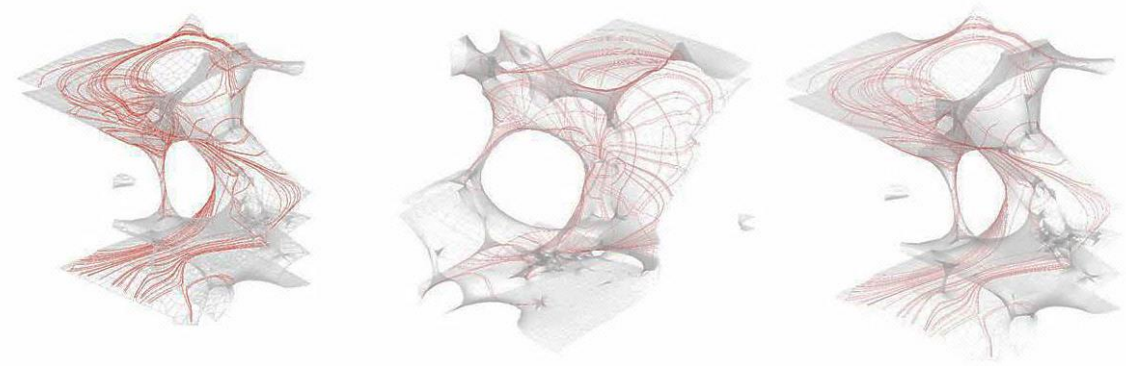

Figure 5. Proto architectural complexes as integrated formal/spatial systems. The result of the lawful interaction of physical forces or organic systems where the shapes are integrated and adapted. 


\section{2-3- The Phase from Prototyping to Adaptive Implementation of the Architectural Body}

The most promising system must be introduced in Phase 3, which is defined exclusively by materializing the digital models developed in Phase 2 by examining and adding material systems and constraints. The kinetic models will be tested at a scale as close as possible to the real intended one, pushing the systems further towards an architectural alliance [32].

The goal is not to explore or test different materials and properties and their limitations, but to explore different biological, mechanical, kinetic or automated systems that facilitate the respective type of transformation.
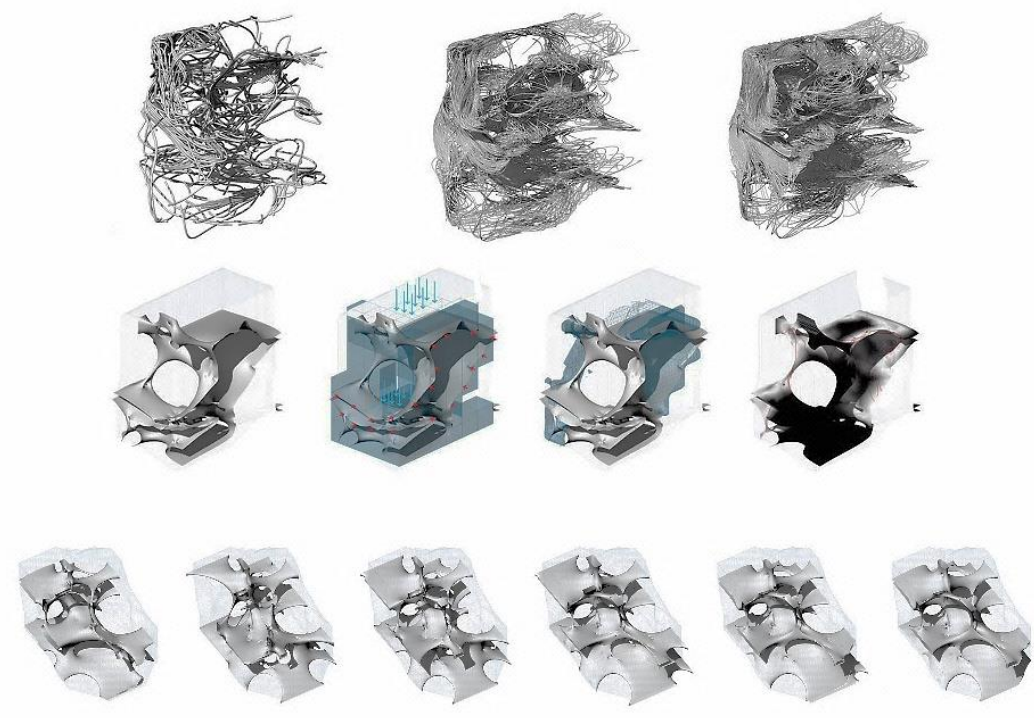

Figure 6. The design system of the prototypes is a combination of multi-systems. Each of them is produced based on the form of another and interact with each other under certain conditions.

The geometry, form and structure of the architectural prototype (proto-architectural) as well as its materials, properties and performance allow first speculations about the scale of the system, the architectural function and the possible location in a (broader) architectural ensemble [33].

Today the abstraction is no longer that of the map, the double, the mirror or the concept. Simulation is no longer that of a territory, a referential being or a substance. It is the creation of a reality without origin or reality through models: a hyperreal. The territory no longer precedes the map and no longer survives it. It is nevertheless the map that precedes the territory - the precession of Simulacra - that creates the territory. It is the real map, not the map, whose traces here and there persist in the deserts, which are no longer those of the Empire, but ours. The desert of the real itself [34].

By applying certain digital frames to animated sequences of analogue research, a new digital material becomes visible and functional. Framing, overlaying and filtering with digital tools becomes a mode for selecting information. Forced distortion and manipulation should be distinguished between excessive information. A creative vision of "what to look for" and "how to look for" became two faces of the same problem in terms of interpretation and reading. In this sense, a more active and important role for representation began to activate the problem of presentation as opposed to representation [35].

However, all the processes in question must be carefully assessed in terms of their ability to design new solutions to specific problems that arise in their scientific field but are also relevant in an architectural context. As these generic processes are not initially able to solve problems outside their domain, they must be appropriate, improved and transferred in the field of architecture [36].

To this end, the intrinsic properties of a generative process will be analysed, abstracted and initially catalogued to be able to speculate on their potential for solving architectural and urban problems. In a next step, the process is described mathematically so that the results can be simulated and reproduced in a simple script or a parametric method.

One of the ideas developed in this particular medium was to present rather than represent actions, problems, solutions and speculations. Animation was then understood not only as representation in time, but also as a way of thinking and understanding the constitution and construction of form within time. A strategy that takes time to construct forms could include more evidence or data, as well as dynamic inputs that are gradually incorporated as new problems and shifts to be solved [37]. 


\section{3- The Potential of the Architectural Body}

Search for guiding principles for a "behaviour-oriented" architecture. Architecture that responds to the human environment has created an old obsession to bring architectural forms to life. Some examples of this research are Karel Honzik's biotechnology: Functional Design and the Plant World (1937); Richard Neutra's Survival through Design (1954); Reyner Banham and François Dallegret's Environment Bubble (1965); Superstudios Microevent / Microenvironment (1972); Markos Novaks Transarchitecture (1995) and Karl Chus Genetic Architecture (2000). Their theoretical assumptions share an understanding of architectural achievements that relate to the ability to reflect and deduce the complexity of the natural organism. Although they come from different knowledge contexts, these assumptions have led to an architectural approach that is closely linked to life and its ability to flow and generate information. Intensity, expansion and potential are three concepts associated with the exponential influence of information on current architectural production. Their respective characteristics have created a fear that no longer arises from the desire to represent life, but from the desire to create it. It is suggested here to review some arguments in relation to the reasons why architecture has always been concerned with the integration of information domains [38].

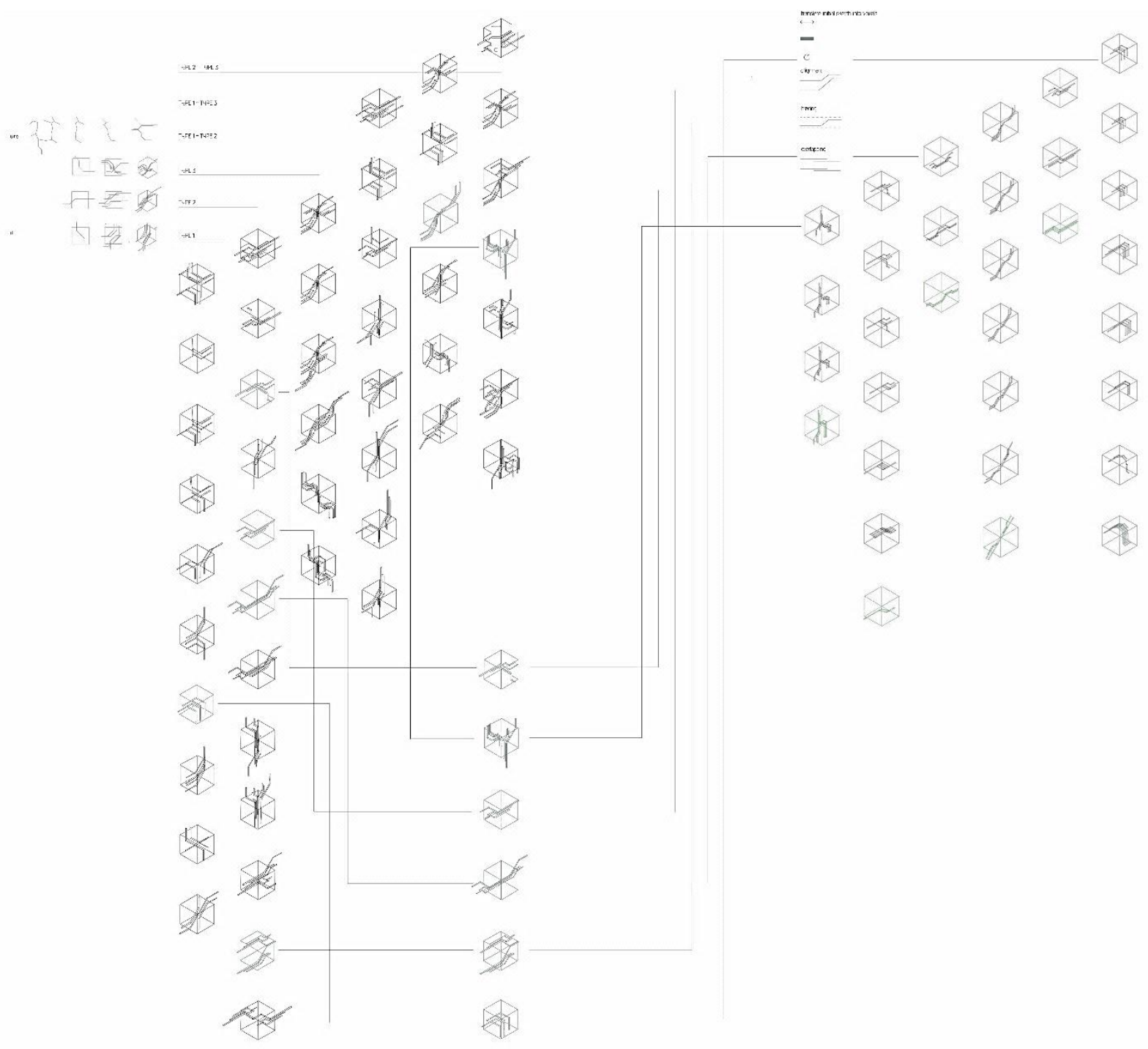

Figure 7. Analogical method that relies on a precise initial investigation of the respective models, objectives and principles.

\section{4- Abstract: Digital Organisms}

With the dramatic development of information theories and related technologies the discipline of architecture has moved from a simple representation of life to the development of systems that ensure the conditions for its creation and sustainability. One of the consequences of this change is the disappearance of the object and its replacement by abstract systems, which are responsible for the creation of "total environments". This "life without objects" triggered numerous projects and researches aimed at developing the environments in which information would be considered. The common currency for all components of the physical or virtual, real - organic or non-organic, living or inert. This analogy Mechanics and electrical services with systems that regulate the living organism is remarkable because it 
suggests that the accumulation of energy functions as diverse as those of climate, wireless and network-based systems implies the disappearance of the form, image and representation of the architectural object [39].

The proto architecture proposes a revision of the linear methods and systems, which are each binary of perception. Architectural objects should no longer be perceived as independent units, but as a unified body, a continuous discursive environment composed of different elements, but capable of interpolating and negotiating their elements by subjecting them to a self-referential process. Evolution and selection guided by the forces acting on them (as an organism) and the functional changes produced are a tribute to this conglomerate of mechanical, electrical and structural systems with their associated requirements and interactions [40]. Here the architectural mass is no longer a living machine, but instead a machine for the literal reproduction of life. The amniotic coating preserves and nourishes the human body and transmits and displays chemical, physical and biological information. It is important here that the house disappears as an object, while the information systems extend in all directions. Proto architecture is not limited to a collection of essential components to support the human body. It is above all a "baroque system" whose existence depends on its ability to collect, link and connect a wealth of information. In other words, the architectural organism is primarily an operational set of compressed data units. This amplified system is also reactive. It involves a multitude of adaptation processes to the external and internal environment of the human body [41]. The environment as a network of infrastructures marks the emergence of a performance design paradigm for an architecture of life, energy and regulated behaviour.[42] Similar to a living organism, the hybrid matter leaves the energy flows, with the organic veins forming a unified system of interconnected and interacting subsystems that efficiently combine to form a whole. Beyond its biological analogy, proto architecture resembles an abstract system of operating devices defined by the behaviours, skills, and knowledge sets that are born and shared. Architecture has finally become an interface for the transmission of energy, sensory and transparent flows. This radical mutation of the architectural object in an abstract system was largely influenced by the emergence of a new perception of the real [43].

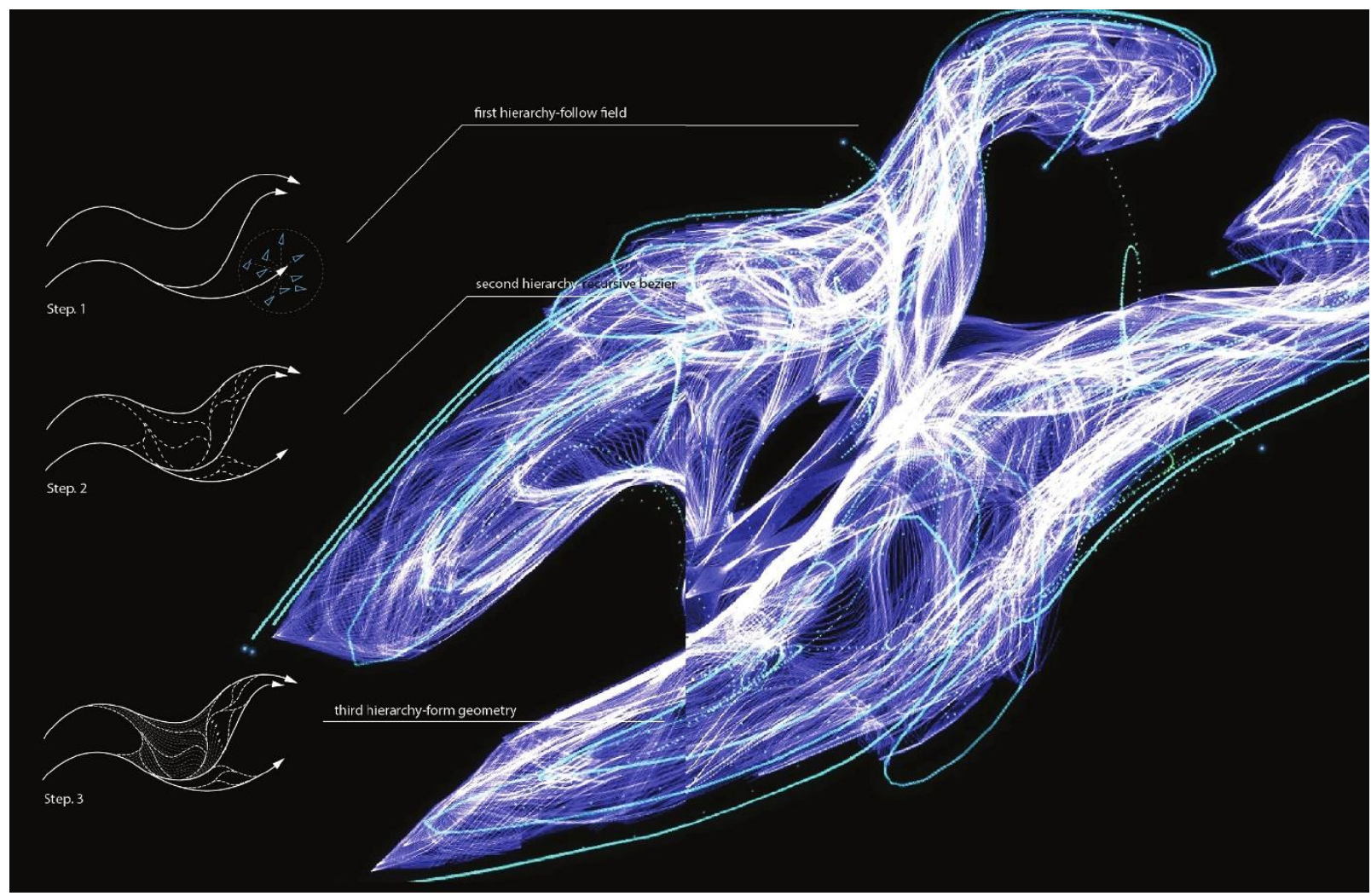

Figure 8. Multi-behaviours systems - the wire keeps changing behaviours when it runs through the space, forming solid surfaces and space frame for the structure part by moving in different patterns.

In the experiment, the network of energy and information that extends to any suitable living space is represented by a Cartesian surface, which of course is not only understood in a physical sense, but also as a visual-verbal metaphor for an ordered and rational distribution of resources [44]. This was presented as a project that represented a critical position in relation to the expansion of abstract networks stimulated by the increasing expansion of information resources. The latter developed a critical model that heralded the emergence of an information economy as a network that reconfigures both production and consumption until its final disappearance: the merged, integrated and unlimited platform of different information processes. With this experiment I tried to describe an anti-ideological architectural organism formed by an abstract machine of naturalized technology in which information penetrates and circulates on the surface [45]. 
For morphogenesis architecture, the shape and matter do not cease to mediate the social and political spheres and is reduced to a concentrated "energy and communication network, an. dimensional model of knowledge. In this sense, the process presents the model of a mental attitude. This is not a three-dimensional model of reality that can be concretized by a simple transposition of the function, but a visual representation of a critical attitude towards (or hope for) design activity, understood as philosophical speculation, as a means [46].

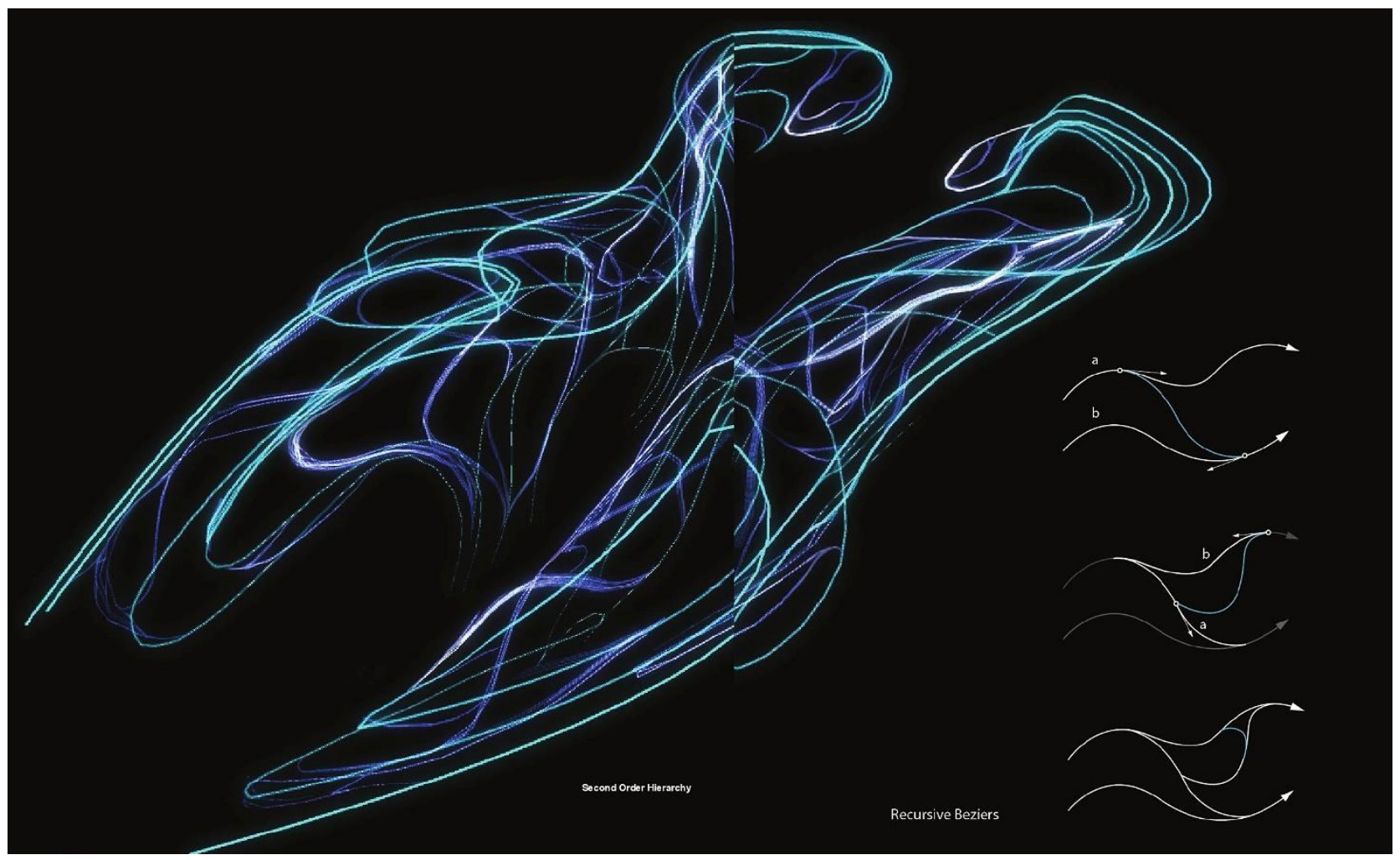

Figure 9. The branching behaviour will continuous recursively and finally generate a whole form of tine recursive curves of continuity and hierarchy. The behaviours are not isolated, they exchange in different situations and are continuous.

\section{5- To Knowledge as Critical Existence}

In this protorealistic environment, the object is replaced by a unified field of flowing information, replacing typology, a spatial representation of the social structure, with a spontaneous, non-conflicting social arrangement [47]. The collective leads to connectivity, the causal event of ubiquitous fiction, the rigid structure of the open system, the border to the infinite horizon. The architectural organism represents a condition. The second critical digital parameter where information networks replace the hierarchical attributes of quantity and quality it marks the emergence of a coding mode underlying the omnipresence of information. It provides an information model in which each component of the system represents a "potential" for a particular event that spills over to the rest of the network [48].

The simulation expresses three main conditions related to the type of information.

- First of all, these critical hybrids recognize the intensity associated with the information flows that penetrate the most in-depth structure of matter;

- Secondly, the expansion of information networks has caused the disappearance of the object, or in other words, its reconfiguration and movement in an unlimited environment of transparent operations;

- Thirdly, the conceptualization of the real in terms of information units implies that the environment and human nature are controlled, directed and managed by mechanisms which, when discovered, contribute to the formation of rules and guiding principles.

These mechanisms form the basis of the laws that govern the space understanding of reality. The striking feature of these mechanisms is their ability to add, integrate and organize large amounts of information in systems that are perceived as increasingly abstract due to their complexity. These information mechanisms represent a reality that is frequently characterized by possibilities, instabilities and probabilities. Approaching our existence in terms of information reveals a world of intensity, expansion and potentiality in the image of hybrid architectural mechanical systems and morphogenesis endless information field [49]. 
The main objective is, therefore, to identify processes by which architectural and urban fields can be effectively structured, modulated and differentiated on a variable scale and which can lead to unpredictable and interesting design results [50]. This is by nature, a heuristic and teleological process in which the characteristics of the architectural results depend on the original configuration. It is also an authentic architectural process, since its purpose is to systematically define and explore a given design space in order to find architectural solutions that are optimized for a given design problem.
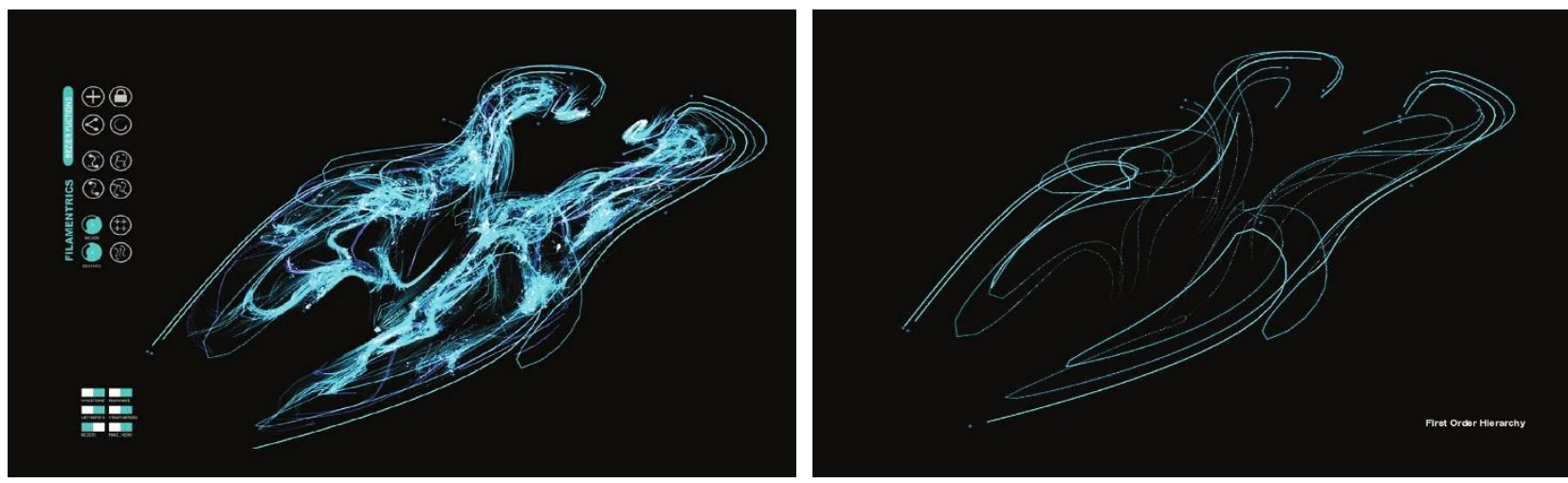

Figure 10. Initially, the first hierarchy of the design system is generated by agents that flow the vector field while affect one another with behaviours of cohesion, separation and alignment.
Figure 11. Secondly, each agent will rad the structure data and branches to generate a curve between the former structure layers, based on the structure information.

The investigation of the morphogenetic potential is one of the most interesting aspects of algorithmic design [51]. The significant difference between generating the form and finding the form should be clearly understood, since algorithmic design aims at exploiting its generative capacity rather than developing a method to inspire form discovery. A systematic variation of intrinsic and extrinsic forces leads to a wide range of possible results, while the range of parameter values determines the ability to produce unexpected results [52].

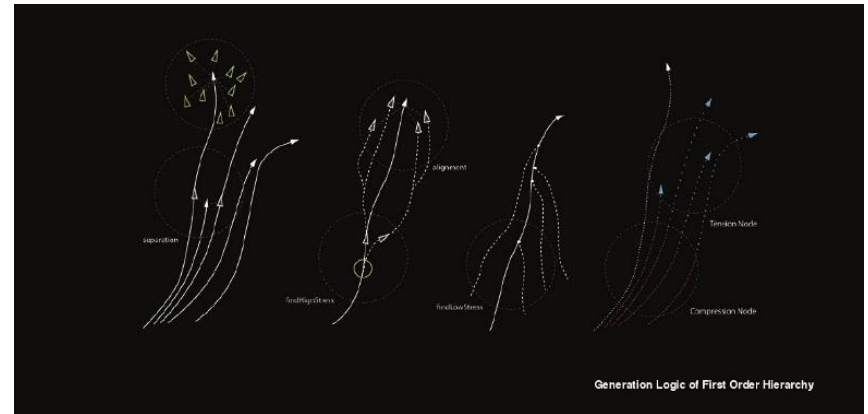

Figure 12. Bezier branches to generate former cohesion.

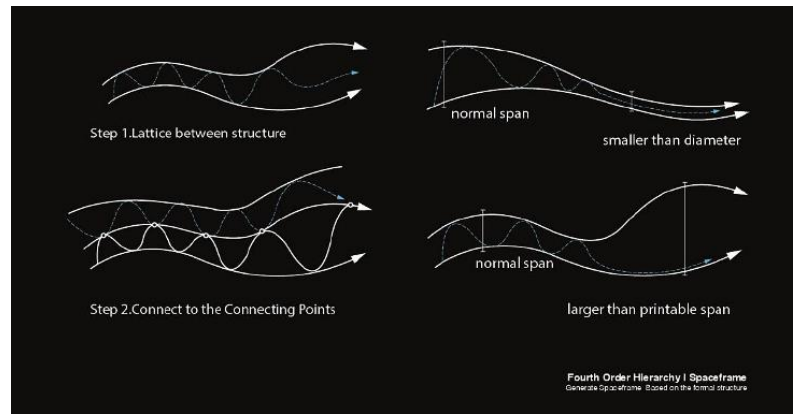

Figure 13. Bezier branches to generate former separation and alignment.

Complex architectural compositions can be perceived as intrinsic to process, propose, apply, analyse and describe complex phenomena and interdisciplinary concepts: Component shapes and structures of dynamic, self-optimised architectural bodies, which automatically consist of geometric systems Mobile phones based on network theory [53].

\section{6- Digital Organisms as a Behavioural Model}

The introduction of information technology triggered this transformation of the studio into a science laboratory. From the very beginning, technological processes aimed to produce models that were increasingly efficient, precise and sensitive. The change in practice does not mean that architecture has become a new science, but that its instruments have become scientific. Based on current practice, these experimental procedures have gradually transformed the fixed and idealized state of the architect into a state that activates behavioural, reactive and adaptive systems [54]. One consequence has been the emergence of architectural models, which today more than ever are associated with the concepts of intensity, expansion and potentiality. Some current debates in the scientific community offer a perspective on how these three concepts can be evaluated and how they influence contemporary architectural production and the perception of reality associated with it [55]. 


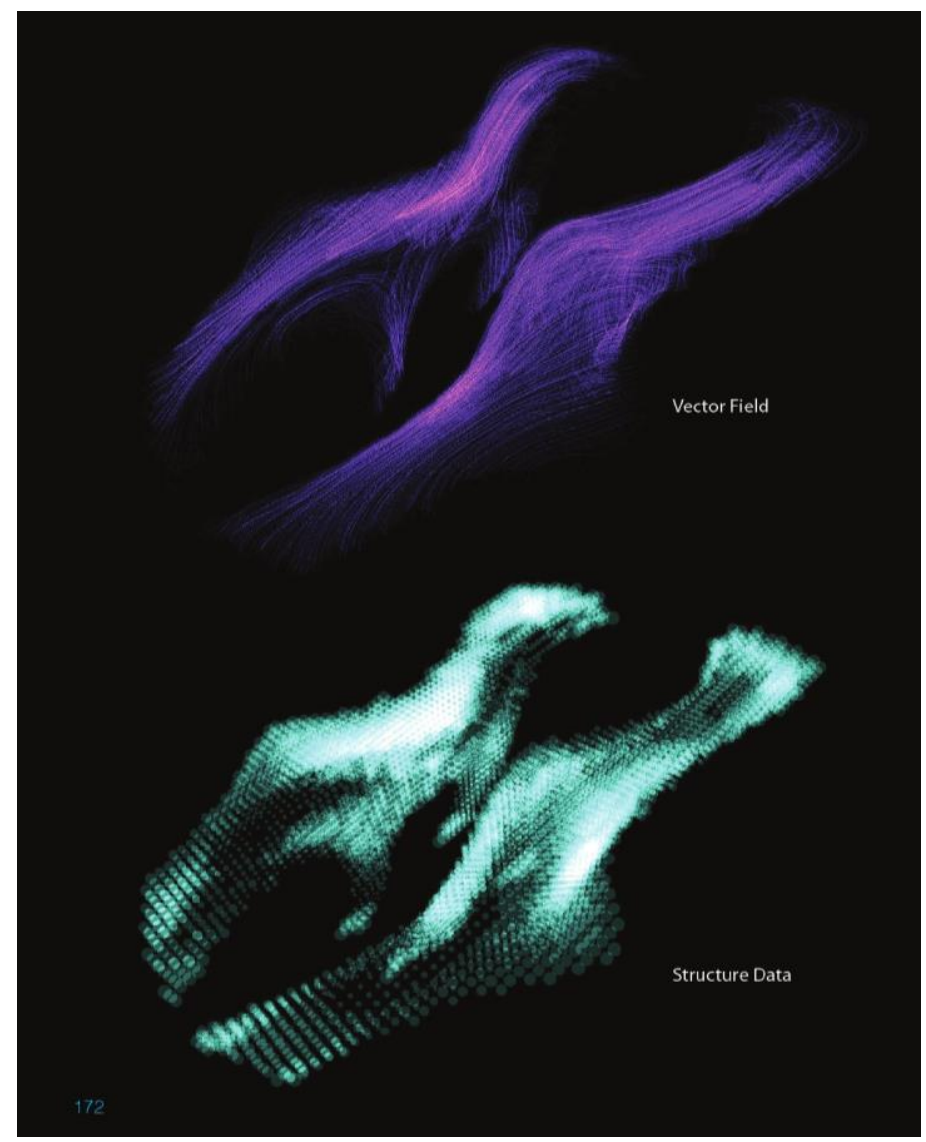

Figure 14. The navigation system can - to a certain extend - re-organize the organizational logic which might be initially implied.

Architecture's ability to transmit and screen information has led to an exponential development of technologies that will take study of space as shape and matter back to the origins of life while advancing a future based on ever faster evolutionary processes. The emergence of new sciences based on the nature of information, such as genomics, biochemistry and computational biology, has accelerated our evolution and adaptation. This acceleration results from the increasing specialisation of technological tools, an excellent economy of death and time. In other terms: of architecture evolution and adaptation [56].

Compared to the long and patient growth of nature, the research points out that technology has shortened time to the limits of human understanding. The technology is seen as a function of the intensity, the intensity of memory and the association of information collected at all levels of reality. This exponential development of technology has changed our perception of the world and in particular its representation. One of the main consequences of this vision is the elimination of the distinction between living and non-living organisms. Science has indeed created a continuum between conscious and unconscious beings, considering that they are made of the same material substance but have a different organisation. While there are noticeable differences between living and living organisms and between conscious and unconscious beings, science also recognises an essential unity between them; a historical agreement, if we consider their processes of prebiotic and biological evolution [57, 58]. It is a question of boundaries, boundaries between architectural space and the environment. These boundaries are increasingly blurred by the mechanical nature of the information reported in kind. For scientists, who are continually researching molecular and atomic interactions, nature really seems to be animated by increasingly automated laws. This model of nature does not predict a posthuman era, as some would argue, but the emergence of a model in which man is no longer idealised and limited to a particular site [59]. In this model, people are more than ever the connected to the world while acknowledging the existence of typical genetic or molecular traces that inorganic matter belongs to humanity and all other sources of organic or organic material. Information has thus limited the limits and transformed a reality into a continuous expansion. A reality formed by abstract and comprehensive mechanisms that organise themselves and continuously recombine.

In response to intense evolutionary technological models and extended shape limits, the experimental models are rooted in a set of laws, the number and yet limitation of which is hugely condensed in terms of information. With the exponential development of technology, laws have indeed generated models that combine both intense and extended operations [60]. These laws tend to be a continuum of guiding principles that develop the macroscale phenomena of our universe and microscale observations of matter. They form an inherently mechanical world, as we find 
everywhere, classical laws such as the principle of relativity or abstract laws such as those of quantum theory. The nature of these laws and mechanisms expresses the potential for the unfolding of the dynamic processes taking place in nature. It is essential that this model of experimental thinking is based on an additional concept. The addition of a variety of principles leads to the emergence of potentials that are combined with the ultimate goal of playing a world that is comprehensively organized. There is no cause and effect, rather a combination of potentials, an addition of all events that can occur outside of a constraint. The potential proto-architectures model is particularly interesting given the nature of the information. With its ability to circulate in all fields of research, the data has increased the confluence of knowledge on a technological platform that is increasingly integrated but reconfigured continuously under the influence of potential parameters [61].

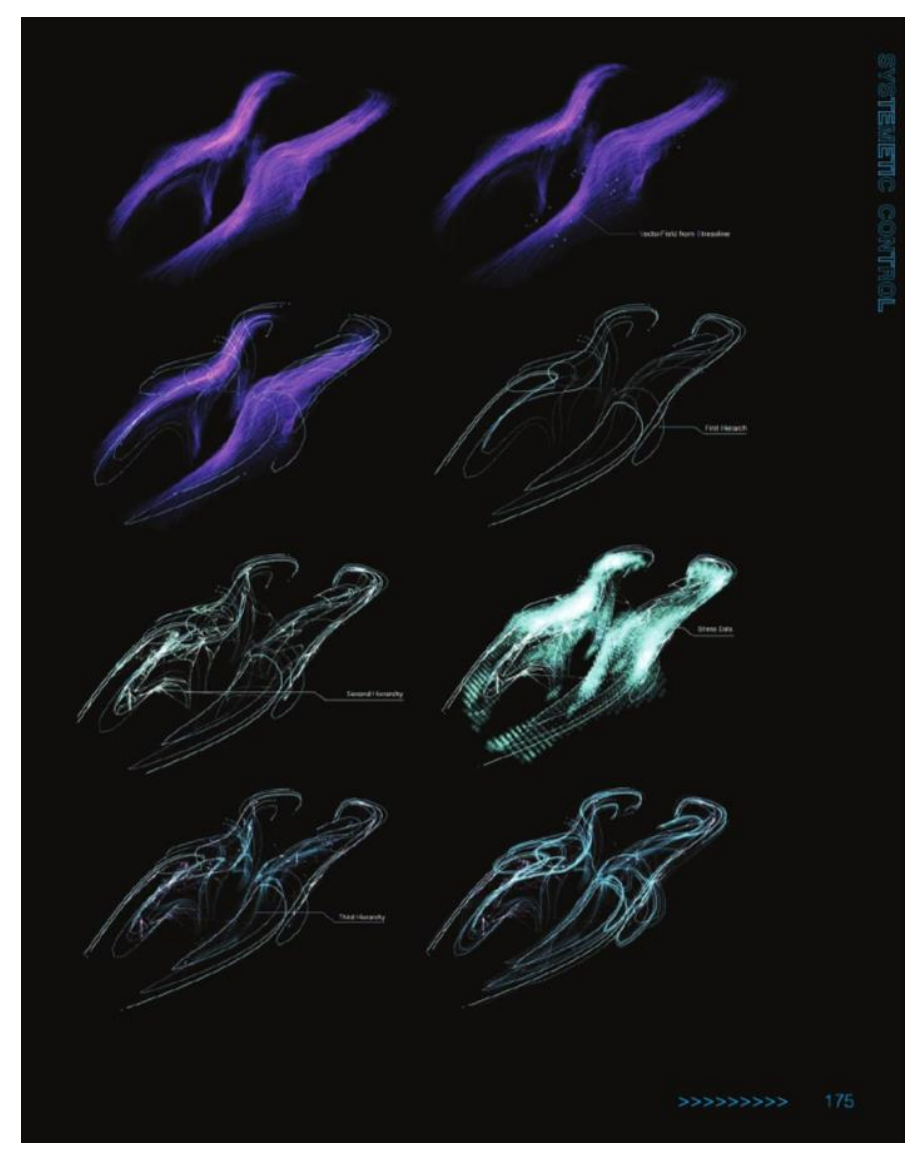

Figure 15. Parallel to typological and organizational developments the study was structured in phases that investigate the relationship between formal spatial organization and programmatic relationships that were taken up to a level of experiential affection.

This computing capacity depends not only on intensive adaptation processes. It also contains an extension condition. Under the pressure of computational tools developed mainly in other fields of knowledge, today's architectural body is no longer autonomous, but is based on a variety of research areas. One of the consequences of this transdisciplinary state is the current proliferation of design activities in emerging fields such as materials and manufacturing research, interactive and immersive means and, most visibly, biologically inspired models. Greg Lynn remarked: "In the near future, it will not be surprising that architects will design molecules in science labs". In other words, the expansion of information and related technologies implies that architecture becomes increasingly porous other areas of knowledge. Its concerns are no longer limited to a specific size, but extend simultaneously to all scales, from the intrinsic structures of the material to the macroscale of environmental phenomena. As a result, the discipline faces a large number of parameters that are retransmitted, processed and resampled by sophisticated computer protocols. Surprisingly, these virtual engines are continuously driven by a wealth of information that blurs the boundaries between organic and inorganic materials, living organisms and living organisms. Architecture has now adopted extension bio-morphogenesis models and created a knowledge continuum that extends to all levels [48].

This continuum of hybridizing architecture has radically changed the nature of practice. With a great variety of information and technologies, architectural unity has evolved from a static to a dynamic state. It now resembles an energy system, which means that its existence depends on adding and assigning parameters, each of which is a potential condition for reconfiguring its intrinsic nature. Technology, in particular, has exponentially increased its ability to add parameters, producing models that are mostly idealistic and are referred to as "emergent". This term is 
often used to describe an architectural entity that expresses a formal complexity generated by increasingly fuzzy computing operations. Nevertheless, the redundant use of this term is not surprising given the current reality, which appears increasingly unstable and changeable.

Over the last 40 years, roughly since the emergence of information science and technology, architecture has undergone a profound change in its status. And yet it remains fascinated by the today's morphogenetic desires of life and its complexity. Given the three concepts of intensity, expansiveness and potentiality that operate in the deepest structures of our informed world, architecture is no longer interested in representing life, but in creating its conditions for evolution, adaptation and duration [62]. The architectural body is now sensitive, mutative and responsive to its existence or, as Peter Sloterdijk puts it, now encompasses the environmental "spheres" of our world.

The configuration of an iterative process, in which the primary sequence is continuously reorganized after the preliminary evaluation of results, becomes an essential problem of the design process once a basic logic has been established, as design research shows the success of the slow construction of a complex system on a relatively simple philosophy.

The systematic exploration and subsequent application of the emergent and non-linear behaviour of the system leads to the emergence of new and unpredictable design models within the design process. In order to expand the repertoire of formal geometries, it is necessary to systematically investigate what contemporary science can contribute to architecture and urbanism beyond the well-known classical canon [63]. The acquisition of complex recurrent geometric models and the underlying mathematical and biological concepts as well as the understanding of emerging fractal, chaotic or self-optimizing systems and their up- and proliferation logic will be used as a source for the experimental development of architectural and urbanistic patterns with high differentiation and varying degrees of density that can accommodate a whole range of emerging hybrid spatial conditions.

\section{7- Conclusion}

Procedural, parametric and generative computer-supported techniques in combination with mass customization and automated fabrication enable holistic manipulation in silico and the subsequent production of increasingly complex architectural arrangements. By automating parts of the design process, computers make it easier to develop design through versioning and gradual adjustment. In recent architectural discourse, these approach to designing have been described as morphogenesis. This paper invites further reflection on the possible meaning of this imported concept in the field of architectural designing. Deriving examples from case-studies, the paper suggests potentials for collaboration and opportunities for bi-directional knowledge transfers.

This research paper has considered existing understandings of morphogenesis in architectural design and biology by discussing examples of cellular, computationally - generated structures, constructed to represent the morphogenesis of a proto architectural organism. The comparison between the arrangements and the respective modes of production in these case-studies demonstrated that semi-living architectural morphogenesis is characterised by complex and flexible mechanisms that can suggest interesting directions for the development of procedural techniques in the architectural domain. Amongst these directions were: 1) implementation of a multi-level hierarchy reflecting functional and structural composition of an architectural arrangement similar to that describing the organisation of a simple biological organisation; 2) implementation of a capability for dynamism in the exchange of signals between organisms; and 3) implementation of another kind of dynamism that can account for topological changes through adaptive structures and kinetic morphologies. Illustrated examples of computational models simulating effects of various control mechanisms in architectural prototypes demonstrated practical effects of selective growth capable of producing kinetic semi-living architectural organism.

\section{8- Data Availability Statement}

The original contributions presented in the study are included in the article, further enquiries can be directed to the corresponding author.

\section{9- Conflict of Interest}

The author declares that there is no conflict of interests regarding the publication of this manuscript. In addition, the ethical issues, including plagiarism, informed consent, misconduct, data fabrication and/or falsification, double publication and/or submission, and redundancies have been completely observed by the authors.

\section{0- References}

[1] Davis, Henry G. "Conservative Surgery, as Exhibited in Remedying Some of the Mechanical Causes That Operate Injuriously Both in Health and Disease." New York, D. Appleton and Company (1867).

[2] Davis, J. "A Closed Loop." (2014). Available online: https://aeon.co/essays/the-feedback-loop-is-a-better-symbol-of-life-thanthe-helix (accessed on August 2020). 
[3] Corucci, Francesco. "Evolutionary Developmental Soft Robotics: Towards Adaptive and Intelligent Soft Machines Following Nature's Approach to Design.” Biosystems \& Biorobotics (September 22, 2016): 111-116. doi:10.1007/978-3-319-46460-2_14.

[4] Corucci, Francesco, Marcello Calisti, Helmut Hauser, and Cecilia Laschi. "Shaping the body to shape the behavior: a more active role of the morphology in the brain-body trade-off." In Proceedings of the 13th European Conference on Artificial Life (ECAL2015), Late Breaking Proceedings, New York, (2015): 7-8.

[5] Corucci, Francesco, Nick Cheney, Hod Lipson, Cecilia Laschi, and Josh Bongard. "Evolving swimming soft-bodied creatures." In ALIFE XV, The Fifteenth International Conference on the Synthesis and Simulation of Living Systems, Late Breaking Proceedings, Cancún 6. (2016a).

[6] Corucci, F., Cheney, N., Lipson, H., Laschi, C., and Bongard, J. "Material properties affect evolution's ability to exploit morphological computation in growing soft-bodied creatures," in ALIFE XV, The Fifteenth International Conference on the Synthesis and Simulation of Living Systems (Cancún: MIT Press), (2016b): 234-241.

[7] Cheney, Nick, Robert MacCurdy, Jeff Clune, and Hod Lipson. "Unshackling evolution: evolving soft robots with multiple materials and a powerful generative encoding." In Proceedings of the 15th Annual Conference on Genetic and Evolutionary Computation, (Amsterdam: ACM), (2013): 167-174.

[8] Ijspeert, A. J. "Biorobotics: Using Robots to Emulate and Investigate Agile Locomotion." Science 346, no. 6206 (October 9 , 2014): 196-203. doi:10.1126/science.1254486.

[9] Hiller, Jonathan, and Hod Lipson. "Automatic Design and Manufacture of Soft Robots.” IEEE Transactions on Robotics 28, no. 2 (April 2012): 457-466. doi:10.1109/tro.2011.2172702.

[10] Hiller, Jonathan, and Hod Lipson. "Dynamic Simulation of Soft Multimaterial 3D-Printed Objects." Soft Robotics 1, no. 1 (March 2014): 88-101. doi:10.1089/soro.2013.0010.

[11] Hiller, J. D., and Lipson, H. "Multi material topological optimization of structures and mechanisms," in Proceedings of the 11th Annual Conference on Genetic and Evolutionary Computation (Montreal, QC: ACM), (2009): 1521-1528.

[12] Nolfi, S., and Floreano, D. Evolutionary Robotics: The Biology, Intelligence, and Technology of Self-Organizing Machines. Cambridge, MA: MIT Press, (2000).

[13] Nolfi, S., O. Miglino, and D. Parisi. "Phenotypic Plasticity in Evolving Neural Networks." Proceedings of PerAc'94. From Perception to Action (1994): 146-157. doi:10.1109/fpa.1994.636092.

[14] Stamps, Judy A. “Individual Differences in Behavioural Plasticities.” Biological Reviews 91, no. 2 (April 10, 2015 ): $534-567$. doi:10.1111/brv.12186.

[15] Wahby, M., Hofstadler, D. N., Heinrich, M. K., Zahadat, P., and Hamann, H. "An evolutionary robotics approach to the control of plant growth and motion: modelling plants and crossing the reality gap," in Self-Adaptive and Self-Organizing Systems (SASO), 2016 IEEE 10th International Conference On (Augsburg: IEEE), (2016): 21-30.

[16] Wahby, M., Soorati, M. D., von Mammen, S., and Hamann, H. "Evolution of controllers for robot-plant bio-hybrids: a simple case study using a model of plant growth and motion," in Proc. of the 25th Workshop on Computational Intelligence (Dortmund: KIT Scientific Publishing), (2015): 67-86.

[17] Hall, B. K. "Evolutionary Developmental Biology." Berlin: Springer Science \& Business Media, (1999).

[18] Gould, S. J. Ontogeny and Phylogeny. Cambridge, MA: Harvard University Press, (1977).

[19] Levi, Paul, and Serge Kernbach. "Symbiotic Multi-Robot Organisms." Cognitive Systems Monographs (2010): 435. doi:10.1007/978-3-642-11692-6.

[20] Zidan, Mohammed A., John Paul Strachan, and Wei D. Lu. "The Future of Electronics Based on Memristive Systems.” Nature Electronics 1, no. 1 (January 2018): 22-29. doi:10.1038/s41928-017-0006-8.

[21] Stanley, Kenneth O. "Compositional Pattern Producing Networks: A Novel Abstraction of Development." Genetic Programming and Evolvable Machines 8, no. 2 (May 10, 2007): 131-162. doi:10.1007/s10710-007-9028-8.

[22] Jakobi, Nick, Phil Husbands, and Inman Harvey. "Noise and the Reality Gap: The Use of Simulation in Evolutionary Robotics.” Lecture Notes in Computer Science (1995): 704-720. doi:10.1007/3-540-59496-5_337.

[23] Ulrich, Y., J. Saragosti, C. K. Tokita, C. E. Tarnita, and D. J. C. Kronauer. "Fitness Benefits and Emergent Division of Labour at the Onset of Group Living.” Nature 560, no. 7720 (August 2018): 635-638. doi:10.1038/s41586-018-0422-6.

[24] Hauser, H., Füchslin, R. M., and Pfeifer, R. (2014). Opinions and Outlooks on Morphological Computation. Zürich. Available online: http://www.merlin.uzh.ch/publication/show/10528 (accessed on August 2020).

[25] Hauser, Helmut, Auke J. Ijspeert, Rudolf M. Füchslin, Rolf Pfeifer, and Wolfgang Maass. "Towards a Theoretical Foundation for Morphological Computation with Compliant Bodies.” Biological Cybernetics 105, no. 5-6 (December 2011): 355-370. doi:10.1007/s00422-012-0471-0. 
[26] Hauser, Helmut. "Resilient Machines through Adaptive Morphology.” Nature Machine Intelligence 1, no. 8 (July 17, 2019): 338-339. doi:10.1038/s42256-019-0076-6.

[27] Weaver, Ian C G, Nadia Cervoni, Frances A Champagne, Ana C D’Alessio, Shakti Sharma, Jonathan R Seckl, Sergiy Dymov, Moshe Szyf, and Michael J Meaney. "Epigenetic Programming by Maternal Behavior.” Nature Neuroscience 7, no. 8 (June 27, 2004): 847-854. doi:10.1038/nn1276.

[28] Stanley, K. O. "Exploiting regularity without development," in Proceedings of the AAAI Fall Symposium on Developmental Systems (Menlo Park, CA: AAAI Press), (2006): 37.

[29]Agrawal, A. A. "Phenotypic Plasticity in the Interactions and Evolution of Species." Science 294, no. 5541 (October 12, 2001 ): 321-326. doi:10.1126/science.1060701.

[30] Russell, S. J., and Norvig, P. Artificial Intelligence: A Modern Approach. Englewood Cliffs, NJ: Prentice Hall, (1995).

[31] McEvoy, M. A., and N. Correll. "Materials That Couple Sensing, Actuation, Computation, and Communication." Science 347, no. 6228 (March 19, 2015): 1261689. doi:10.1126/science.1261689.

[32]Frei, Regina, and Giovanna Marzo Serugendo. “The Future of Complexity Engineering.” Open Engineering 2, no. 2 (January 1, 2012). doi:10.2478/s13531-011-0071-0.

[33] Laskowski, Kate L., Carolina Doran, David Bierbach, Jens Krause, and Max Wolf. "Naturally Clonal Vertebrates Are an Untapped Resource in Ecology and Evolution Research.” Nature Ecology \& Evolution 3, no. 2 (January 28, 2019): 161-169. doi:10.1038/s41559-018-0775-0.

[34] Groothuis, Ton G.G., and Fritz Trillmich. "Unfolding Personalities: The Importance of Studying Ontogeny." Developmental Psychobiology 53, no. 6 (August 22, 2011): 641-655. doi:10.1002/dev.20574.

[35] McNamara, Kenneth J. "Heterochrony: The Evolution of Development." Evolution: Education and Outreach 5, no. 2 (June 2012): 203-218. doi:10.1007/s12052-012-0420-3.

[36] Wehner, Michael, Ryan L. Truby, Daniel J. Fitzgerald, Bobak Mosadegh, George M. Whitesides, Jennifer A. Lewis, and Robert J. Wood. “An Integrated Design and Fabrication Strategy for Entirely Soft, Autonomous Robots.” Nature 536, no. 7617 (August 2016): 451-455. doi:10.1038/nature19100.

[37] Kriegman, S., Walker, S., Shah, D. S., Kramer-Bottiglio, R., and Bongard, J. “Automated Shapeshifting for Function Recovery in Damaged Robots," in Robotics: Science and Systems XV (Messe Freiburg), (2019). Available online: https://arxiv.org/pdf/1905.09264.pdf (accessed on 22 May 2020).

[38] Brooks, R. A. “Artificial life and real robots,” In Proceedings of the First European Conference on Artificial Life (Cambridge, MA: MIT Press), (1992): 3-10.

[39] Sims, K. "Evolving virtual creatures," In Proceedings of the 21st Annual Conference on Computer Graphics and Interactive Techniques (Orlando, FL: ACM), (1994): 15-22.

[40] Moczek, Armin P., Sonia Sultan, Susan Foster, Cris Ledón-Rettig, Ian Dworkin, H. Fred Nijhout, Ehab Abouheif, and David W. Pfennig. "The Role of Developmental Plasticity in Evolutionary Innovation." Proceedings of the Royal Society B: Biological Sciences 278, no. 1719 (June 15, 2011): 2705-2713. doi:10.1098/rspb.2011.0971.

[41] Kelly, Scott A., Tami M. Panhuis, and Andrew M. Stoehr. "Phenotypic Plasticity: Molecular Mechanisms and Adaptive Significance.” Comprehensive Physiology (April 2012): 1417-1439. doi:10.1002/cphy.c110008.

[42] amazine, S., Deneubourg, J.-L., Franks, N. R., Sneyd, J., Theraulaz, G., and Bonabeau, E. "Self-Organization in Biological Systems. Princeton," NJ: Princeton University Press, (2001).

[43] Dall, Sasha R. X., Alison M. Bell, Daniel I. Bolnick, and Francis L. W. Ratnieks. "An Evolutionary Ecology of Individual Differences." Edited by Andrew Sih. Ecology Letters 15, no. 10 (August 16, 2012): 1189-1198. doi:10.1111/j.14610248.2012.01846.x.

[44] Dorigo, Marco, Vito Trianni, Erol Şahin, Roderich Groß, Thomas H. Labella, Gianluca Baldassarre, Stefano Nolfi et al. "Evolving self-organizing behaviors for a swarm-bot." Autonomous Robots 17, no. 2-3 (2004): 223-245. doi:10.1023/B:AURO.0000033973.24945.f3.

[45] Wang, Frank Z., Ling Li, Luping Shi, Huaqiang Wu, and Leon O. Chua. “Ф Memristor: Real Memristor Found.” Journal of Applied Physics 125, no. 5 (February 7, 2019): 054504. doi:10.1063/1.5042281.

[46] Scholz, Christian, Michael Engel, and Thorsten Pöschel. "Rotating Robots Move Collectively and Self-Organize.” Nature Communications 9, no. 1 (March 2, 2018). doi:10.1038/s41467-018-03154-7.

[47] Dingemanse, Niels J., Anahita J.N. Kazem, Denis Réale, and Jonathan Wright. "Behavioural Reaction Norms: Animal Personality Meets Individual Plasticity." Trends in Ecology \& Evolution 25, no. 2 (February 2010): 81-89. doi:10.1016/j.tree.2009.07.013. 
[48] White, P.J., K. Kopanski, and H. Lipson. “Stochastic Self-Reconfigurable Cellular Robotics.” IEEE International Conference on Robotics and Automation, 2004. Proceedings. ICRA’04. 2004 (2004): 2888-2893. doi:10.1109/robot.2004.1307499.

[49] Schmidt, Michael, and Hod Lipson. "Age-Fitness Pareto Optimization.” Genetic and Evolutionary Computation (October 20, 2010): 129-146. doi:10.1007/978-1-4419-7747-2_8.

[50] Rus, Daniela, and Michael T. Tolley. "Design, Fabrication and Control of Soft Robots.” Nature 521, no. 7553 (May 2015 ): 467-475. doi:10.1038/nature14543.

[51] Kennedy, Patrick, Gemma Baron, Bitao Qiu, Dalial Freitak, Heikki Helanterä, Edmund R. Hunt, Fabio Manfredini, et al. "Deconstructing Superorganisms and Societies to Address Big Questions in Biology." Trends in Ecology \& Evolution 32, no. 11 (November 2017): 861-872. doi:10.1016/j.tree.2017.08.004.

[52] Whiten, Andrew, Francisco J. Ayala, Marcus W. Feldman, and Kevin N. Laland. "The Extension of Biology through Culture." Proceedings of the National Academy of Sciences 114, no. 30 (July 24, 2017): 7775-7781. doi:10.1073/pnas.1707630114.

[53] Lipson, Hod, and Jordan B. Pollack. “Automatic Design and Manufacture of Robotic Lifeforms.” Nature 406, no. 6799 (August 2000): 974-978. doi:10.1038/35023115.

[54] Walter, W. Grey. “An Imitation of Life.” Scientific American 182, no. 5 (May 1950): $42-45$. doi:10.1038/scientificamerican0550-42.

[55] Schmickl, Thomas, Ronald Thenius, Christoph Moslinger, Jon Timmis, Andy Tyrrell, Mark Read, James Hilder, et al. "CoCoRo -- The Self-Aware Underwater Swarm." 2011 Fifth IEEE Conference on Self-Adaptive and Self-Organizing Systems Workshops (October 2011): 120-126. doi:10.1109/sasow.2011.11.

[56] Wolf, Jason B., Edmund D. Brodie III, and Allen J. Moore. "Interacting Phenotypes and the Evolutionary Process. II. Selection Resulting from Social Interactions." The American Naturalist 153, no. 3 (March 1999): 254-266. doi:10.1086/303168.

[57] Veenstra, F., Faina, A., Støy, K., and Risi, S. "Evolving self-organizing simulated plant-inspired robots," in Workshop on Methods for Self-Organizing Distributed Systems, Laubusch, (2015).

[58] Veenstra, F., Faina, A., Stoy, K., and Risi, S. "Generating artificial plant morphologies for function and aesthetics through evolving L-systems," in Proceedings of the Artificial Life Conference (Cancùn: MIT Press), (2016).

[59] Bağbanc1, Bilal Muhammed. "Dynamic Identification of an Early 20th Century Civil Architectural Building." Civil Engineering Journal 6, no. 4 (April 1, 2020): 670-678. doi:10.28991/cej-2020-03091499.

[60] Sadeghi, A, A Mondini, E Del Dottore, V Mattoli, L Beccai, S Taccola, C Lucarotti, M Totaro, and B Mazzolai. "A PlantInspired Robot with Soft Differential Bending Capabilities." Bioinspiration \& Biomimetics 12, no. 1 (December 20, 2016): 015001. doi:10.1088/1748-3190/12/1/015001.

[61] Divband Soorati, Mohammad, Mary Katherine Heinrich, Javad Ghofrani, Payam Zahadat, and Heiko Hamann. "Photomorphogenesis for Robot Self-Assembly: Adaptivity, Collective Decision-Making, and Self-Repair." Bioinspiration \& Biomimetics 14, no. 5 (July 12, 2019): 056006. doi:10.1088/1748-3190/ab2958.

[62] Pandolfi, Camilla, and Dario Izzo. "Biomimetics on Seed Dispersal: Survey and Insights for Space Exploration." Bioinspiration \& Biomimetics 8, no. 2 (May 7, 2013): 025003. doi:10.1088/1748-3182/8/2/025003.

[63] Winfield, Alan F. T., and Mehmet Dincer Erbas. "On Embodied Memetic Evolution and the Emergence of Behavioural Traditions in Robots." Memetic Computing 3, no. 4 (July 8, 2011): 261-270. doi:10.1007/s12293-011-0063-x. 